\title{
Persistent Pulmonary Hypertension of the Newborn: Analysis of 181 cases over one Year
}

\author{
Nurun Nahar Fatema \\ Combined Military hospital, Dhaka
}

\begin{abstract}
:
Keywords:

Congenital heart

disease,

Pulmonary

hypertension.

Background: Persistent pulmonary hypertension (PPHN) is the persistence of the high pulmonary arterial pressure after birth which is a characteristic of the fetal circulation. PPHN is associated with substantial infant mortality and morbidity. Various treatment protocol are used according to the need of the patient. This study aimed to see the spectrum of the disease and its outcome in Bangladesh.

Methods: 181 newborn babies were diagnosed as persistent pulmonary hypertension or persistent fetal circulation. After establishing diagnosis, all cases were kept in NICU and various management plans were prescribed according to necessity of a specific case. Repeat echocardiography was done after 72 hours to see the response of treatment. Residual congenital cardiac lesions were managed later on.

Results: Most of the patient (69.06\%) was diagnosed at first week of life. Minimum age of the patient was 1 day and maximum age 23 days. Among 181 cases 109 (66.22\%) were male and 72(39.77\%) were female. Associated congenital lesions like atrial septal defect was found in $52(28.72 \%)$ cases, patent ductus arteriosus (PDA) was found in $14(7.73 \%)$ cases and ventricular septal defect (VSD) was found in $2(1.10 \%)$ cases. Combination of ASD \& PDA was found in $75(44.33 \%)$ cases. Systolic pulmonary artery pressure was more than $60 \mathrm{mmHg}$ in 103 (56.91\%) cases, more than $50 \mathrm{mmHg}$ in $53(29.28 \%)$ cases and more than $30 \mathrm{mmHg}$ in $25(13.81 \%)$ cases. Most of the patient (100\%) received high flow oxygen therapy along with anti failure (66.30\%) and sildenafil $98(54.14 \%)$ therapy as per requirement of the patient. Complete cure was achieved in $95.58 \%$ cases and mortality was only $1.10 \%$.
\end{abstract}

Conclusion: These data showed a very impressive outcome of the PPHN in Bangladeshi Newborn even with minimally invasive management.

(Cardiovasc. j. 2018; 11(1): 17-22)

\section{Introduction:}

Persistent fetal circulation (PFC), also known as persistent pulmonary hypertension of the newborn, was first described as "unripe births of mankind" by William Harvey in $1628 .{ }^{1}$ Novelo et al. presented a paper that also described postnatal persistence of fetal circulatory patterns. Lind and Wegelius demonstrated that "the foramen ovale has either failed to close or reopened" in asphyxiated infants. ${ }^{2,3}$ Survival of a newborn at birth requires an immediate and sustained fall in pulmonary vascular resistance from its elevated level in utero to a low resistance, high flow circulation after delivery. This rapid drop allows eight fold increase of blood flow to the lungs that enable it to exchange gases. Some infants do not achieve or sustain this change of normal decrease in pulmonary vascular resistance at birth leading to severe respiratory distress and hypoxemia which is known as persistent pulmonary hypertension of newborn. (PPHN). ${ }^{4}$ This is usually seen in newborn with respiratory distress syndrome, overwhelming sepsis, meconium aspiration syndrome, intrauterine hypoxemia or ischemia and or neonatal hypoxemia or ischemia. ${ }^{5} \mathrm{PPHN}$ is a major clinical syndrome contributing significantly to high morbidity or mortality in both full term and premature neonates. ${ }^{6,7}$ In modern neonatal intensive care unit, anticipation and early treatment of PFC and its complications in sick newborns with good results are common events. So severe PFC are only seen in rare occasions. The aim of this study was to see spectrum of the disease and its outcome in Bangladesh.

\section{Methods:}

This is a retrospective study carried out in the non-invasive cardiac laboratory of a tertiary hospital from February 2016 to January 2017.

Address of Correspondence: Dr. Nurun Nahar Fatema, Combined Military Hospital, Dhaka, Bangladesh.

E mail: colfatema@hotmail.com 
Out of total 13245 cases of color Doppler echocardiography in this period, 181 cases were diagnosed as persistent pulmonary hypertension of newborn or persistent fetal circulation. After diagnosis, all the outpatient cases were admitted to the neonatal intensive care unit (NICU) for specific management. Some of the cases were already in NICU for their existing illness.

Echocardiography parameters which were noted in each case were:

- Measurement of pulmonary artery systolic and Diastolic pressure

- Right ventricular dimension and thickness.

- Presence of atrial Septal defect or patent Ductus Arteriosus and direction of shunt through them.

- Presence of any other significant shunt.

Those who had right to left shunt were considered as persistent fetal circulation. Patients who had pulmonary hypertension associated with significant congenital lesions like large ventricular Septal defects, Aorto pulmonary window, Atrioventricular septal defects or other complex lesions were not included in the study. Birth history, antenatal history of mother like history of hypertension or diabetes, History of TORCH Infection, Gestational age of the baby, birth weight, APGAR

score, mode of delivery were recorded. Treatment protocols used for the patients were as follows:

First line treatment:

a) High flow oxygen therapy for 72 hours.

b) Tab. Cardopril/ Enalepril (Pulmonary vasodilator).

c) Inj. Frusemide (Preload and afterload reduction).

d) Inj. Digoxin (RV dysfunction).

2nd line treatment:

a) Tab. Sildenafil (pulmonary vasodilator).

b) High frequency ventilation with alkalinization to treat profound hypoxemia and acidosis.

c) Inotropic support with Dopamine and/or Dobutamine in low dose.

d) Inj. Indomethacine or Syp: Ibuprofen or Paracetamol to close the ductus.

e) Tab. Bosentan for pulmonary vasodfilatation.

f) Inj. Magnesium sulphate $\left(\mathrm{MgSO}_{4}\right)$.

g) Inj. Prostaglandin.

3rd line management:

a) Inhaled Nitric Oxide (INO) and ECMO (not available in Bangladesh).

\section{Results:}

Table-I

Age Distribution of Patients at diagnosis (N=181).

\begin{tabular}{|c|c|c|c|c|c|c|c|}
\hline \multicolumn{2}{|c|}{$1^{\text {st }}$ Week } & \multicolumn{2}{|c|}{$2^{\text {nd }}$ Week } & \multicolumn{2}{|c|}{$3^{\text {rd }}$ Week } & \multicolumn{2}{|c|}{ 4rth Week } \\
\hline Number & Percentage & Number & Percentage & Number & Percentage & Number & Percentage \\
\hline 125 & $69.06 \%$ & 37 & $0.44 \%$ & 09 & $4.97 \%$ & 10 & $5.52 \%$ \\
\hline
\end{tabular}

Most of the cases are (69.06\%) diagnosed at first week of life, least (5.52\%) diagnosed at 4rth week.

Table-II

Sex Distribution of Patients (N=181).

\begin{tabular}{lccc}
\hline Male & & \multicolumn{2}{c}{ Female } \\
Number & Percentage & Number & Percentage \\
\hline 109 & $60.22 \%$ & 72 & $39.77 \%$ \\
\hline
\end{tabular}


Table-III

Disease Distribution of cases $(N=181)$.

\begin{tabular}{lcc}
\hline Disease & Number & Percentage \\
\hline PPHN & 40 & $22.09 \%$ \\
PPHN+ ASD & 52 & $28.72 \%$ \\
PPHN+ ASD+PDA & 55 & $30.38 \%$ \\
PPHN+ PFO & 04 & $2.20 \%$ \\
PPHN+PDA & 14 & $7.73 \%$ \\
PPHN+PDA+ PFO & 07 & $3.86 \%$ \\
PPHN+ASD+PDA+VSD & 02 & $1.10 \%$ \\
PPHN+LPA STENOSIS & 04 & $1.48 \%$ \\
PPHN+MVP & 03 & $1.48 \%$ \\
\hline
\end{tabular}

Out of 181 cases, $109(66.22 \%)$ were male and 72 (39.77\%) were female. Associated disease along with PPHN. Forty (22.09\%) cases had PPHN only. Atrial Septal Defect (ASD) was seen in 52 (28.72\%) cases. only Patent ductus arteriosus was found in $14(7.75 \%)$ cases and ventricular Septal Defect (VSD) along with ASD and PDA was found in $02(1.10 \%)$ cases. (Combination of ASD \& PDA was found in 73 (40.33\%) cases.

\section{Table-IV}

Distribution of Patients according to severity of pulmonary hypertension $(N=181)$.

\begin{tabular}{lcc}
\hline Pulmonary hypertension & Number & Percentage \\
\hline Mild (30-50 $\mathrm{mm}$ of $\mathrm{Hg})$ & 17 & $9.39 \%$ \\
Moderate (> 50-60 mm of $\mathrm{Hg})$ & 42 & $23.20 \%$ \\
Severe (> 60 mm of Hg) & 122 & $67.40 \%$ \\
\hline
\end{tabular}

Severity of pulmonary hypertension classified by echocardiography. Twenty five (13.81\%) cases had mild pulmonary hypertension, 53 (29.28\%) cases had moderate pulmonary hypertension and 103 (56.90\%) cases had severe pulmonary hypertension.

Table-V

Echocardiographic findings of Patients ( $N=181)$.

\begin{tabular}{llcc}
\hline Parameter & Findings & Number & Percentage \\
\hline Aortic Valve & Decreased & 50 & $27.62 \%$ \\
Velocity & Increased & 00 & $00 \%$ \\
& Normal & 131 & $72.37 \%$ \\
RV Volume & Decreased & 00 & 00 \\
& Increased & 171 & $94.47 \%$ \\
& Normal & 10 & $5.52 \%$ \\
LV Volume & Decreased & 08 & $4.41 \%$ \\
& Increased & 20 & $11.09 \%$ \\
& Normal & 161 & $88.95 \%$ \\
\hline
\end{tabular}

Echocardiography findings of Patient with Pulmonary hypertension. RV volume was increased in $171(94.47 \%)$ cases LV volume was increased in $20(11.04 \%)$ cases. Aortic valve velocity was decreased in $50(27.62 \%)$ cases.

\section{Table-VI}

Modality of treatment offered to the patients $(N=181)$.

\begin{tabular}{lcc}
\hline Treatments modalities & Number & Percentage \\
\hline High flow oxygen therapy & 181 & $100 \%$ \\
Anti failure treatment & 120 & $66.29 \%$ \\
Pulmonary vasodilator & 98 & $54.14 \%$ \\
(sildenafil) & 08 & $4.41 \%$ \\
High frequency ventilator & 00 & 00 \\
Extra corporeal membrane & 00 & 00 \\
$\begin{array}{l}\text { Oxygenation (ECMO) } \\
\text { Inhaled Nitric Oxide (INO2) }\end{array}$ & 00 & 00 \\
$\begin{array}{l}\text { Inotropes (Dopamine, } \\
\text { Dobutamine) }\end{array}$ & 25 & $13.81 \%$ \\
$\begin{array}{l}\text { Inj.Indomethacin/Ibuprofan/ } \\
\text { Paracetamol }\end{array}$ & 08 & $4.41 \%$ \\
$\begin{array}{l}\text { Correction of acidosis or } \\
\text { alkalosis }\end{array}$ & 62 & $34.25 \%$ \\
\hline
\end{tabular}

Treatment protocol offered in neonatal intensive care unit: High flow oxygen therapy was offered to 181 (100\%) cases, anti-failure with Frusemide, Captopril, and Digitalis were prescribed in all 120 $(66.29 \%)$ cases. Tablet Sildenafil was given to 98 (54.14\%) cases. High frequency ventilation was given to $8(4.41 \%)$ cases. Dopamine and Dobutamine was given in 25 (13.81\%) cases. Inj. indomethacin was used to close significant PDA in 08 (4.41\%) cases. Corrections of acidosis or alkalosis were indicated in62 (34.25\%) cases. ECMO and inhaled nitric oxide (NO) was not advised.

\section{Table-VII}

Outcome of treatment $(N=181)$.

\begin{tabular}{lcc}
\hline Variables & Number & Percentage \\
\hline Cured & 173 & $95.58 \%$ \\
Expired & 02 & $1.10 \%$ \\
Develop IPAH & 06 & $3.31 \%$ \\
Left with ASD, PDA\& VSD & 71 & $39.22 \%$ \\
\hline
\end{tabular}

Out of 181 cases, 173 (95.58\%) cases cured completely, 2 (1.10\%) cases expired, 6 (3.31\%) cases progressed to idiopathic pulmonary arterial hypertension, and 71(39.22\%) cases later required ASD and $\mathrm{PDA}$ device closure 
Table-VIII

Follow up of patient $(N=181)$.

\begin{tabular}{|c|c|c|c|c|c|}
\hline \multirow[t]{2}{*}{ Follow up } & \multicolumn{2}{|c|}{ Number } & \multirow[t]{2}{*}{ Total } & \multicolumn{2}{|c|}{ Percentage } \\
\hline & Male & Female & & Male & Female \\
\hline 1st Follow up after 72 hours & 109 & 72 & 181 & $60.22 \%$ & $39.77 \%$ \\
\hline $2^{\text {nd }}$ Follow up after one month & 25 & 21 & 46 & $54.34 \%$ & $45.65 \%$ \\
\hline
\end{tabular}

Hundred percent patient attended first follow up of echocardiography as they were hospitalized but $2^{\text {nd }}$ follow up after one month was attended by $54.34 \%$ male and $45.65 \%$ female babies.

\section{Discussion:}

Persistent fetal circulation (PFC) also known as persistent pulmonary hypertension of the newborn is defined as persistence of right to left atrial or ductal shunting or both in the presence of elevated right ventricular pressure..$^{1-3}$ Mean pulmonary artery pressure at rest is $>25 \mathrm{~mm}$ of $\mathrm{Hg}$ in such situation with normal pulmonary capillary wedge pressure. PPHN results from abnormal vascular remodeling. Increased pulmonary arterial resistance and exaggerated vascular activity. It is relatively a rare condition which is seen in newborn with respiratory distress syndrome, septicemia, meconium aspiration syndrome, diaphragmatic hernia or perinatal asphyxia or ischemia. ${ }^{8,9}$ In utero the fetus derives its oxygen and nutrients from placenta through the umbilical vein. Most of this blood bypasses the liver and enter into right atrium (RA). From RA blood enter into left atrium through foramen ovale and subsequently pumped into Aorta. Negligible amount of blood that enter into right ventricle from RA is subsequently pumped into Aorta through ductus arteriosus bypassing lung as pulmonary vascular resistance is very high in fetus. So fetal lung receive only $8 \%$ of ventricular output. Because a lower PVR generally promotes functional closure of the ductus and foramen ovale while a high PVR encourages PFC, it is useful to know which substances increase and which substances decrease PVR. ${ }^{10-12}$ Factors known to lower PVR include oxygen, nitric oxide, prostacyclin, prostaglandins $\mathrm{E}_{2}$ and $\mathrm{D}_{2}$, adenosine, magnesium, bradykinins, atrial natriuretic factor, alkalosis, histamine, acetylcholine, beta-adrenergic stimulation and potassium channel activation. Factors that increase PVR are hypoxia, acidosis, endothelin-1, leukotrienes, thromboxanes, platelet activating factors, prostaglandin $\mathrm{F}_{2}$-alpha, alpha-adrenergic stimulation and calcium channel activation ${ }^{13-16}$. Thus, it is important to recognize clinical conditions that affect PVR and to treat them appropriately. Mechanisms that contribute to high pulmonary vascular resistances in the fetus are: Low oxygen tension b) relatively low basal production of vasodilator products (PGI, nitric oxide) c) increased production of vasoconstrictors (endothelin and leukotrienes) and altered smooth muscle activity. During last trimester, the fetal pulmonary circulation becomes progressively responsive to vasoactive Stimuli. ${ }^{8-10}$ Immediately after birth, pulmonary artery pressure falls and blood flow increases in response to birth related stimuli. Those includes establishment of air liquid interface, rhythmic lung distension, increased oxygen tension and altered production of vasoactive substances like nitric oxide and prostaglandin. ${ }^{4,5}$ Mechanisms that lead to failure of fall in pulmonary resistance at birth include a) exposure to chronic or acute hypoxia at birth, b) chronic hypoxia in utero. c) Entrance of meconium into the airways d) septicemia e) cardiac malformations and others. ${ }^{10,11}$ The syndrome occurs in 1-2 per 1000 live births typically in full term babies. It occurs more commonly in male. Most of the cases of PFC landed to complete recovery or death. ${ }^{16}$ Occasionally, there may be long term sequelae such as chronic lung disease, cerebral infarction resulting in specific motor and/ or cognitive deficits, and sensorineural hearing loss. ${ }^{17}$ An association with sudden infant death syndrome has also been suggested.PFC can be primary or secondary to other factors. Any problems or situations can result in idiopathic PFC, including hypoxia, acidosis, hypothermia, hypoglycemia etc. Secondary PFC is most commonly seen in infants with lung diseases, the most common cause being meconium aspiration. The resulting hypoxia and acidosis cause pulmonary vasoconstriction and increased rightsided pressures. Other common causes are diaphragmatic hernia, hyaline membrane disease, 
sepsis syndrome and pulmonary thromboembolism. These babies usually present with cyanosis, respiratory distress, oxygen dependency, low cardiac output stapes, differential cyanosis etc. Differential cyanosis is due to the difference in oxygen content in preductal and postductal blood, and is relatively specific for PFC. A positive partial pressure of arterial oxygen $\left(\mathrm{PaO}_{2}\right)$ gradient greater than 15 $\mathrm{mmHg}$ between the right radial artery and the descending aorta blood suggests PFC, but it may be absent in some cases. ${ }^{17}$ The hyperoxia test is also positive, so PFC is suspected particularly when these conditions cannot be corrected by improved ventilation.

Chest X-ray may be normal or there may be oligaemic lung fields, congenital diaphragmatic hernia etc.

Echocardiography is the most sensitive and specific investigation which help to confirm the diagnosis of PPHN. 18,19 Parameters used in our settings are:

a. Right ventricular dimension and thickness

b. Left ventricular dimension and output

c. Direction of shunt through holes in atrial, ventricular or ductus level

d. Calculation of pulmonary artery systolic and diastolic pressure from tricuspid and pulmonary regurgitation jet.

Infants with PFC are very sensitive to their environment and tend to be extremely unstable. They are, in general, mechanically ventilated, sedated and often paralyzed with muscle relaxants. ${ }^{20,21}$ Therefore, procedures such as suctioning, changing endotracheal tubes, bathing and repositioning should be kept to a minimum. Early treatment with surfactant prevents the development of PFC in cases with lung issues. Controlled hyperventilation has been used to decrease PVR by making the blood more alkaline. The administration of cardiotonic drugs should be reserved for infants in whom myocardial dysfunction and/or persistent hypotension is documented. Exogenous, inhaled nitric oxide at low doses causes potent, sustained and selective pulmonary vasodilation. ${ }^{22,23}$

The patient who had decreased left ventricular size and aortic velocity had increased likelihood to need ECMO support, INO, high frequency ventilation etc. In our study only 08 cases has smaller left ventricular end diastolic areas (Table V) and these cases required high frequency ventilation.

Ventricular volume overload and high calculated pulmonary artery systolic pressure, few with right to left shunting through ASD or PDA and they were treated successfully with high flow nasal and head box oxygen therapy along with administration of pulmonary vasodilator drugs (Table VI). In our study most of the cases were cured (95.58\%)with treatment described in (Table VII).Only 6.65\% Patient developed idiopathic pulmonary arterial hypertension and $2.15 \%$ patient expired (Table VII).In a study conducted in children's hospital of Philadelphia, out of 63 neonates $95 \%$ survived, 14\%required ECMO, 52\% required $\mathrm{HFV}, 67 \%$ INO and $35 \%$ required mechanical ventilationl2. This study showed most of the patient required expensive and invasive treatment modalities for ultimate cure but in our study we achieved high rate of cure without using any expensive and invasive treatment. Most of our cases have PPHN and severe forms of PFC were only seen in isolated occasion now. Outcome of treatment at the end of therapy was assessed by doing echocardiography at 72 hours and documented (Table VIII).Treatment protocol formulated and used for PPHN in our neonatal intensive care unit is cheap, affordable and effective which was proved from our low mortality rate.

\section{Conclusion:}

Respiratory failure is a leading cause of death in newborn, most common cause of which is PPHN. This condition is reversible but can cause very severe and unrelenting respiratory failure and death if remain untreated Recent advancement in diagnostic procedure and treatment expanded the scope for survival in these.

Conflict of Interest - None.

\section{References:}

1. Harvey WA. Exercitatio Anatomica de Motu Cordiset Sanguinis in Animali bus The Classics of Medicine Library; Birmingham, Alabama: 1978. (The Keynes English Translation of 1928) 
2. Novelo S, Lason RL, Bouchard F. Un nouveau syndrome avec cyanosecongenitale: La persistence du canal arterial avec hypertension pulmonaire. Ler Congres Mondial de Cardiolegie; Paris: 1950.

3. Lind J, Wegelius C. Changes in the circulation at birth. Acta Paediatr 1952; 42: 495-496.

4. Abman SH. Persistent pulmonary hypertension of Newborn: Pathophysiology and treatment. Pulmonary hypertension summer 2006; 5 (2) : 22 - 30.

5. Cornfield DN, Reeves HL, Tolarova S. Oxygen Causes fetal pulmonary vasodilation through activation of a calcium dependent potassium channel. Proc Natl Acad Sci 1996; 93: 8089-8094.

6. D'Cunha C, Sankaran K. Persistent Fetal circulation. Paediatric child Health 2001; 6 (10): 744-750.

7. Levin DL, Heymenn MA, Kiterman JA. Persistent pulmonary hypertension of newborn. J Pediatr 1976; 89: 626-633.

8. Kinsella JP, Abman SH. Recent development in the pathophysiology and treatment of PPHN . J Pediatr 1995; 126: 853 - 864 .

9. D'cunha C, Shankaran K. Persistent fetal circulation. Paediatr Child Health 2001; 6 (10):744-750.

10. Rasanen J, Wood DC, Debbs RH, Cohon J, Weiner S, Huhta JC. Reactivity of the human fetal pulmonary circulation to maternal hyper oxygenation increases during the second half of pregnancy. A randomized study. Circulation 1998; 97: $257-262$.

11. Rudloph AM, Heymann MA, Lewis All. Physiology and pharmacology of the pulmonary circulation in the fetus and newborn. In : Hodson w. Ed. Development of the lung. NY: Marcel- Dekk, 1997:497-523.

12. Morin FC, Egan EA, Ferguson W, Lundgren CEG. Development of pulmonary vascular response to oxygen. AMJ Physiol 1988; 254: 542- 546.

13. Abman SH, Shanley PF, Accurso FJ. Failure of postnatal adaptation of the pulmonary circulation after chronic intrauterine pulmonary hypertension in fetal lambs. J Clin Invest 1989; 83: 1849-1858.

14. Koren G, Nordeng H. SSRIs and persistent pulmonary hypertension of the newborn (Editorial) BMJ 012; 344: d7642.15.

15. Beck R. Chronic lung disease following hypocapneic alkalosis for persistent pulmonary hypertension. J Pediatr 1985; 106 : 527-528. [PubMed]

16. Klesh KW, Murphy TF, Scher MS, Buchanan DE, Maxwell $\mathrm{EP}$, Guthrie RD. Cerebral infarction in persistent pulmonary hypertension of the newborn. Am J Dis Child 1987; 141: 852-857. [PubMed]

17. Ballard RA, Leonard CH. Developmental follow-up of infants with persistent pulmonary hypertension of the newborn. Clin Perinatol 1984; 11: 737-744. [PubMed]

18. Naulty CM, Weiss IP, Herer GR. Sensorineural hearing loss in survivors of persistent fetal circulation (PFC). Clin Res 198432918A (Abst).

19. Vesselinova-Jenkins CK. Model of persistent fetal circulation and sudden infant death syndrome (SIDS) Lancet 1980; ii: 831-4. [PubMed

20. Peterson AL, Deatman S, Frommelt MA, Mussatto K, Frommelt PC. Correction of echocardiographic marker and therapy in persistent pulmonary hypertension of newborn. Pediatr Cardiol 2009; 30 (2); 160-165.

21. Fatema NN, Khan MR, Kabir MS. Persistent pulmonary hypertension of newborn; analysis of 93 cases over one year. Bangladesh Journal of Cardiology 2012; 4 (1):55.

22. Drummond W. Use of cardiotonic therapy in the management of infants with persistent pulmonary hypertension of the newborn. Clin Perinatol 1984; 11:715728. [PubMed]

23. Morin F, Stenmark K. Persistent pulmonary hypertension of the newborn. Am J Resp Crit Care Med 1995; 151: 20102032. [PubMed]

24. Kinsella JP, Neish SR, Ivy DD, Shaffer E, Abman SH. Clinical responses to prolonged treatment of persistent pulmonary hypertension of the newborn with low doses of inhaled nitric oxide. J Pediatr 1993; 123:103-108. [PubMed] 